\title{
On a novel method to synthesize POSS-based hybrids: An example of the preparation of TPU based system
}

\author{
O. Monticelli ${ }^{1}$, A. Fina ${ }^{2}$, D. Cavallo ${ }^{3}$, E. Gioffredi ${ }^{2}$, G. Delprato ${ }^{1}$ \\ ${ }^{1}$ Dipartimento di Chimica e Chimica Industriale, Università di Genova, Via Dodecaneso, 31, 16146 Genova, Italy \\ ${ }^{2}$ Dipartimento di Scienza Applicata e Tecnologia, Politecnico di Torino-sede di Alessandria, viale Teresa Michel, 5, 15121 \\ Alessandria, Italy \\ ${ }^{3}$ Department of Mechanical Engineering, Eindhoven University of Technology, P.O. Box 513, 5600 MB Eindhoven, The \\ Netherlands
}

Received 4 July 2013; accepted in revised form 9 August 2013

\begin{abstract}
A novel method to prepare polymer/polyhedral oligomeric silsesquioxanes (POSS) hybrids by melt reactive blending is proposed in this paper, by the controlled polymer chain scission and reaction of chain ends with functional silsesquioxanes. Application to thermoplastic polyurethanes (TPU) is addressed, taking advantage of the polyurethane chain scission equilibrium reaction, leading to the formation of highly reactive isocyanate and hydroxyl chain ends. Despite the isocyanate chemistry has been widely studied for the preparation of polymer/POSS hybrids by in situ copolymerisation, the exploitation of similar chemical processes in an industrially viable and environmental friendly melt blending process is currently an open research field. In this work, the reaction in the molten state of dihydroxyl-functionalised POSS with the polyurethane chain is demonstrated to produce a TPU/POSS hybrids. The effect of POSS concentration on nanomorphology, thermal properties and surface properties is studied, showing significant changes compared to pristine TPU. In particular, an increase of glass transition temperature is observed in the presence of reactive POSS ( $\Delta T$ up to about $10^{\circ} \mathrm{C}$ in the presence of $10 \mathrm{wt} \%$ loading). Furthermore, an increase of surface water wettability, evidenced by the decrease of water contact angle from $95^{\circ}$ for pristine TPU to $70^{\circ}$ in TPU containing $10 \mathrm{wt} \%$ of reactive POSS, is found.
\end{abstract}

Keywords: nanocomposites, POSS, TPU, $T_{\mathrm{g}}$, reaction blending

\section{Introduction}

Polyhedral oligomeric silsesquioxanes (POSS) have been used as an interesting class of precursors for the synthesis of molecularly designed organic/ inorganic hybrids [1]. POSS bridges the gap between fillers and monomers in modifying macromolecules mobility and thus the reinforcement of polymer materials. Indeed, POSS are organic/inorganic molecules, with a size of approximately 1 to $3 \mathrm{~nm}$, with a general formula of $\left(\mathrm{RSiO}_{1,5}\right) \mathrm{n}$ where $\mathrm{R}$ is hydrogen or an organic group, such as alkyl, aryl or any of their derivatives $[2,3]$. The size of the pendant POSS cage is comparable to the dimensions of the linear polymer, enabling POSS to control the motions of the chains at the molecular level with enhanced benefits while retaining the processability and mechanical properties of the pristine polymer [1]. From a general point of view, the incorporation of the thermally robust POSS moiety into organic polymers was found to modify drastically the thermal properties of the polymer supplying improved thermal stability to the polymer matrix [4, 5], also allowing the tailoring of the polymer glass transition temperature by tuning the POSS concentration [6]. Moreover, incorporation of POSS molecules is responsible for modifications of the mechanical

\footnotetext{
*Corresponding author, e-mail: orietta.monticelli@unige.it (C) BME-PT
} 
properties $[7,8]$ as well as reduction in both flammability and rate of heat release during combustion [9]. The preparation of POSS/polymer systems has been accomplished following different approaches. In particular, in situ copolymerization of POSS to produce hybrid organic-inorganic polymers with pendent POSS groups has been widely studied both for thermoplastics and thermosets [10-12]. On the other hand, melt blending was also widely studied, this process being very appealing, economic and environmentally friendly $[13,14]$. Nevertheless, the dispersion of POSS into polymers by simple melt mixing is not obvious and requires the strict control of the balance between polymer-POSS interactions and POSS-POSS self-interactions.

Solution blending $[15,16]$ and vapour phase grafting [17] have also been attempted to incorporate POSS into a polymer matrix. Recently, some work has been also carried out on the preparation of polymer/POSS systems by melt reactive blending techniques [18]. Chemical reactions between POSS and polymer chains in the molten state can indeed be taken into advantage to promote molecular dispersion of POSS [19]. Nevertheless, the above approach can only be applied to polymer matrices carrying reactive side groups capable of reacting with silsesquioxane molecules. On this ground, the generalization of the reactive melting approach to polymers which does not carry reactive side groups is indeed of great interest.

The innovative approach, proposed in the present work, is based on the reaction between silsesquioxane molecules and the macromolecule functional groups, which are formed directly during the melt blending process through a controlled scission of the polymer matrix.

In order to employ chain scission to generate reactive functions to be exploited for further reactions during melt blending, very controlled statistical chain scission should be obtained. This is possible in principle with different polymers, including polyamides, polyesters and thermoplastic polyurethanes. The latter class of polymers has been chosen due to the properties of polyurethanes and the intrinsic reactivity of isocyanate groups.

POSS hybrids based on polyurethane (PU) have been previously prepared by in situ polymerization. Indeed, synthesis of amphiphilic telechelic oligourethanes with terminal POSS groups through reaction of monoisocyanate substituted POSS and oligo (oxyethylene diol) and investigation of the structure of such polymers in solution were reported [20,21]. Interestingly, chemical reaction of monoisocyanate substituted POSS fragments with polyamidoamine of a dendritic nature (polyamidoamine PAMAM dendrimers) leads to the formation of several organoinorganic core-shell type nano-hybrids [22]. Application of dihydroxy-containing POSS with isocyanate pre-polymers can yield linear segmented PU with POSS fragments as a side group of the hard segments [23-28]. By analogy with this method, diamino-POSS was used to prepare nanostructured PU-POSS hybrid aqueous dispersions [29]. As demonstrated, the reaction of the silanol groups of an open cage POSS with isocyanate moieties gave segmented PU with POSS incorporated in the polymer main chain as a part of the hard segment [30]. Cross-linked PU were also synthesized through the reaction of octafunctional POSS with either isocyanate [31] or amine groups. [32]

Few papers reported on the preparation of composites based on PU by melt blending. Bourbigot et al. [33] described the reaction to fire of composites based on TPU containing $10 \mathrm{wt} \%$ of POSS prepared in a Brabender-type mixer.

In this work, a novel method to prepare PU/POSS hybrids directly by melt blending is explored: controlled chain scission of a commercial thermoplastic PU (TPU) is carried out to obtain the formation of functional groups potentially capable of reacting with diol-functionalised polyhedral oligomeric silsesquioxane molecules.

\section{Experimental}

\subsection{Materials}

Octaisobutyl POSS (referred to as oib-POSS in the following) and trans-cyclohexanediolisobutyl POSS (referred to as POSS-OH in the following) were purchased from Hybrid Plastics (Hattiesburg, MS, USA) as crystalline powders and used as received. Chemical structures for oib-POSS $(M=873.6 \mathrm{~g} / \mathrm{mol})$ and POSS-OH $(M=959.7 \mathrm{~g} / \mathrm{mol})$ are reported in Figure 1.

The polyurethane used (Elastollan 1185A), kindly supplied by BASF (Germany), was a polyetherbased thermoplastic PU.

\subsection{TPU/POSS system preparation}

Before accomplishing the hybrid preparation, the polymer matrix was dried overnight at $110^{\circ} \mathrm{C}$. TPU 


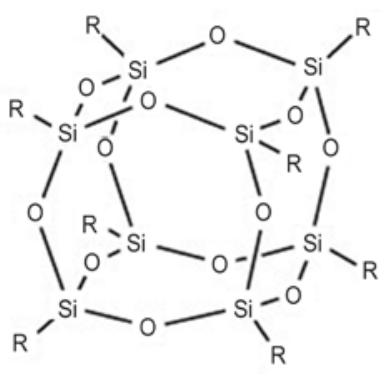

a)

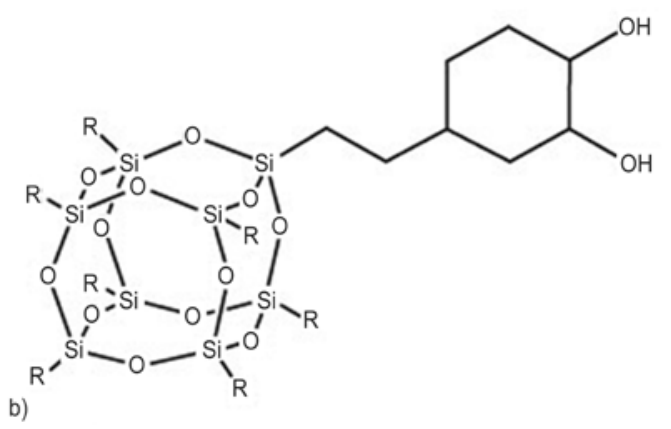

Figure 1. (a) Octaisobutyl POSS (oib-POSS), (b) transcyclohexanediolisobutyl POSS (POSS-OH)

was added to the glass reactor, namely a laboratory internal mixer provided with a mechanical stirrer (Heidolth, type RZR1), which was connected to a vacuum line and evacuated for $30 \mathrm{~min}$ at $100^{\circ} \mathrm{C}$. Then, the reactor was purged with helium for $30 \mathrm{~min}$. The above operations were repeated at least three times, to be sure to avoid humidity to come in contact with the reagents.

The reactor was placed in an oil bath at $220^{\circ} \mathrm{C}$ and when the polymer was completely molten, POSS was added under inert atmosphere. TPU/POSS systems were prepared by mixing, under inert atmosphere, the neat polymer and POSS, both octaisobutyl and trans-cyclohexanediolisobutyl POSS, at various concentrations, from 2 to $20 \mathrm{wt} \%$, using a mixing time of $10 \mathrm{~min}$.

Neat TPU was processed and characterised under the same conditions, as reference material. Materials are identified in the text with the format polymer/POSS type(concentration); as: TPU/POSS$\mathrm{OH}(10)$.

In order to evaluate the reaction yield after melt blending, all solid samples were broken into small pieces and unreacted POSS was removed by Soxhlet extraction with hexane for $48 \mathrm{~h}$. The grafting yield was calculated by weighting composite samples before and after the above treatment.

\subsection{Characterization}

Fourier Transform-Infrared Spectroscopy (FT-IR) spectra were recorded by a Bruker IFS66 spectrometer. The samples were scanned in the range 400 $4000 \mathrm{~cm}^{-1}$ with nitrogen purge.

Differential scanning calorimetry (DSC) was performed under a continuous nitrogen purge on a Mettler (Mettler, Columbus, OH, USA) calorimetric apparatus, model TC10A. Both calibrations of heat flow and temperature were based on a run in which one standard sample (indium) was heated through its melting point. Samples having a mass between 2.5 and $6 \mathrm{mg}$ were used. Data were gathered using a scan rate of $10^{\circ} \mathrm{C} / \mathrm{min}$.

Transmission electron microscopy analyses were performed with a high-resolution equipment JEOL 2010 (Tokyo, Japan). The measurements were carried out using an accelerating voltage of $200 \mathrm{kV}$. Ultrathin sections of about $100 \mathrm{~nm}$ thick were cut with a Power TOMEX microtome equipped with a diamond knife and placed on a 200-mesh copper grid and stained with ruthenium tetraoxide to obtain sufficient phase contrast.

Contact angle measurements were performed at room temperature with an Erma G-1 contact angle meter using pure water as probe liquid.

\section{Results and discussion}

\subsection{Study of the polymer matrix thermal behavior}

This work has been preliminarily focused on the study of TPU thermal behavior, the approach for the hybrid polymer/POSS synthesis being based on a controlled polymer matrix decomposition which allows to create reactive groups, potentially capable of reacting with silsesquioxane molecules. The mechanisms of thermal decomposition of polyurethanes were reviewed by Chattopadhyay and Webster [34]. Despite a number of possible concurrent reactions is possible, urethane bond scission (Figure 2), is typically obtained as a first step, at relatively low temperatures $\left(180-250^{\circ} \mathrm{C}\right)$, depending on the PU structure [34].

$$
\mathrm{R}-\mathrm{NH}-\mathrm{C}-\mathrm{O}-\mathrm{CH}_{2}-\mathrm{CH}_{2}-\mathrm{R}^{\prime} \rightleftarrows \mathrm{RNCO}+\mathrm{HO}-\mathrm{CH}_{2}-\mathrm{CH}_{2}-\mathrm{R}^{\prime}
$$

Figure 2. Thermal degradation mechanism of the urethane segment by depolymerisation 
During thermal decomposition of PU, this first decomposition step is followed by further scission of the hard and soft segments, leading to a number of secondary decomposition products. However, secondary decomposition steps are much slower and thus require significantly higher temperature to occur. Therefore, it is possible in principle to induce a limited extent of chain scission without reaching the conditions of extensive decomposition and volatilisation of the polymer, thus allowing to produce isocyanate groups during melt mixing.

Thermogravimetric measurements, carried out in heating ramp showed no detectable weight loss until $260^{\circ} \mathrm{C}$, supporting no significant volatilisation of polymer during melt blending.

IR spectra of our TPU and that of the polymer treated at $200^{\circ} \mathrm{C}$ are given in Figure 3 .

The FT-IR spectrum of the neat TPU presents a characteristic band at $3328 \mathrm{~cm}^{-1}$, which corresponds to the stretching vibration of $\mathrm{N}-\mathrm{H}$ group [35]. The bands at 2956 and $2870 \mathrm{~cm}^{-1}$ can be attributed to asymmetric and symmetric stretching of $\mathrm{CH}_{2}$ group [36]. The shoulder at $1730 \mathrm{~cm}^{-1}$ characterizes $\mathrm{C}=\mathrm{O}$ stretching vibrations in ester structure, while the peak at $1705 \mathrm{~cm}^{-1}$ corresponds to $\mathrm{C}=\mathrm{O}$ stretching vibration in the urethane bond (amide I band). The absorbance at $1531 \mathrm{~cm}^{-1}$ in the IR spectrum of TPU could be attributed to the coupling of $\mathrm{N}-\mathrm{H}$ bending vibration with $\mathrm{C}-\mathrm{N}$ stretching vibration in the $-\mathrm{C}-\mathrm{NH}$ group (amide II band) $[35,36]$. The weaker vibration band at $1314 \mathrm{~cm}^{-1}$

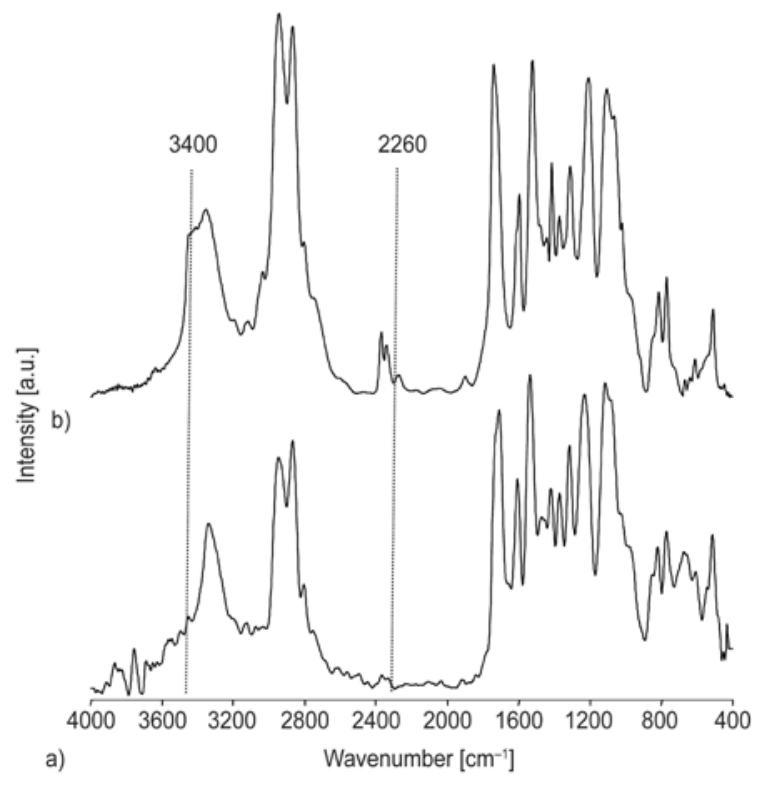

Figure 3. FTIR spectra of (a) TPU and (b) TPU at $200^{\circ} \mathrm{C}$ (inert atmosphere) corresponds to the combination between $\mathrm{N}-\mathrm{H}$ bending vibration and $\mathrm{C}-\mathrm{N}$ stretching vibration (amide III band) [35]. The region 1300-1100 $\mathrm{cm}^{-1}$ is characteristic to $\mathrm{C}-\mathrm{O}$ stretching vibrations in esters. The skeletal vibration of $\mathrm{C}=\mathrm{C}$ in the aromatic ring from $1597 \mathrm{~cm}^{-1}$ can be coupled with the absorbance from $814 \mathrm{~cm}^{-1}$, the last being characteristic to $\mathrm{C}-\mathrm{H}$ out of plane bending vibration in 1,4disubstituted aromatic ring [37]. While the IR spectra of the samples treated below $200^{\circ} \mathrm{C}$ turn out to be similar to that of the neat TPU, the polymer, which underwent a thermal treatment at $200^{\circ} \mathrm{C}$ online in the IR cell, shows some differences. Indeed, the band between 3600 and $3200 \mathrm{~cm}^{-1}$ is modified, with the appearance of a shoulder at $3400 \mathrm{~cm}^{-1}$, while a new peak at ca. $2260 \mathrm{~cm}^{-1}$ appears. The former band can be assigned to hydroxyl group, whereas the one at higher wavenumbers to isocyanate group formation [35].

Moreover, the stability of the polymer matrix molecular mass, evaluated by viscosity measurements, further supports the above mechanism, the relative viscosity of the pristine TPU and that of the treated sample being equivalent, namely 1.42 and 1.41 , respectively.

These findings suggest that the occurrence of the chain scission reaction reported in Figure 2 is indeed an equilibrium reaction, which is weakly shifted towards the dissociated form when increasing the temperature. Therefore, in the presence of POSS$\mathrm{OH}$, reaction of isocyanate groups with POSS is possible, also depending on the reactivity ratio between POSS moieties and OH-terminated polymer chains.

\subsection{Study of hybrid systems characteristics}

The polymer matrix was contacted with various amounts of an hydroxyl-functionalized POSS (from 2 to $20 \mathrm{wt} \%$ ) and, as a comparison, with $10 \mathrm{wt} \%$ of isobutyl-POSS at $200^{\circ} \mathrm{C}$. Up to $10 \mathrm{wt} \%$ the hybrids turned out to be homogeneous, whereas the sample blend containing $20 \mathrm{wt} \%$ of POSS-OH (TPU/POSS$\mathrm{OH}(20))$ clearly showed two separated phases at the micron scale. In the case of the sample based on isobutyl-POSS, (TPU/oib-POSS(10)), a solvent capable to swell the polymer and completely solubilize POSS molecules was found to extract the POSS entirely, evidencing the efficiency of the experimental procedure for the extraction of unbound silsesquioxanes. Conversely, for TPU/POSS(10), 


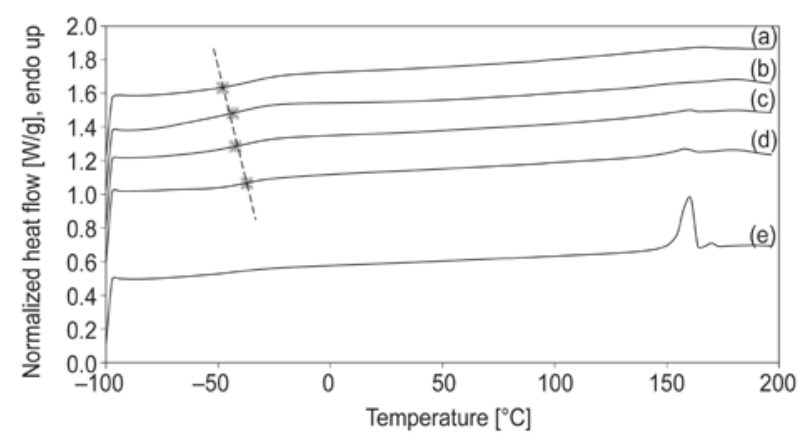

Figure 4. DSC traces on heating for: (a) TPU $\left(T_{\mathrm{g}}=-48^{\circ} \mathrm{C}\right)$, (b) TPU/POSS-OH(2) $\left(T_{\mathrm{g}}=-43^{\circ} \mathrm{C}\right)$, (c) TPU/ POSS-OH $(5)\left(T_{\mathrm{g}}=-42^{\circ} \mathrm{C}\right)$, (d) TPU/POSS-OH(10) $\left(T_{\mathrm{g}}=-37^{\circ} \mathrm{C}\right)$, (e) POSS-OH

containing $10 \mathrm{wt} \%$ of POSS-OH (soluble in the same solvent), the extraction treatment seems to eliminate only a fraction of the silsesquioxane mixed with the polymer, which is only $20 \%$ of the total POSS content for TPU/POSS-OH(10). Moreover, in the case of TPU/POSS-OH(5) and TPU/POSS$\mathrm{OH}(2)$ the reaction yield is almost $100 \%$. This demonstrates the covalent bonding of POSS-OH to the TPU chain, evidencing that the reactivity of the silsesquioxane is essential for the formation of a hybrid system.

DSC traces of the different TPU/POSS-OH prepared are given in Figure 4, in comparison with pristine TPU and POSS-OH.

The pristine TPU displays a glass transition at $-48^{\circ} \mathrm{C}$, which is typical for this kind of polymer. By analyzing these results, it comes out that a modification of $T_{\mathrm{g}}$ with respect to that of the neat polymer matrix occurs even for the samples with low concentration of POSS-OH (TPU/POSS-OH(2)). In particular, the TPU/POSS-OH sample $T_{\mathrm{g}}$ increases with increasing the silsesquioxane content, while in the case of TPU/oib-POSS(10), namely the system containing the unreactive POSS, the glass transition temperature remains constant. This behavior might

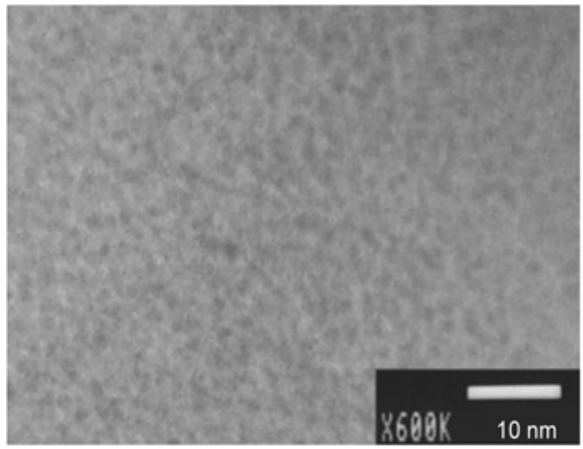

a) be related to the scarce interaction between the isobutyl-POSS and the polymer matrix, as demonstrated by yield data.

Taking into account the literature findings [38-40], it is possible to interpret the increase of the glass transition temperature of our TPU/POSS systems on the basis of hindrance of polymer chain mobility induced by the presence of relatively bulky POSS cages. Moreover, for our polymer matrix, which consists of a hard and a soft segment phase, as reported in the literature [29], the relevant increase of $T_{\mathrm{g}}$ at low temperature with increasing the POSS content can be ascribed to the portioning of silsesquioxane molecules into the soft segment phase.

It is worth to underline the presence of a very small peak in the DSC trace of TPU/POSS-OH(5), which is more evident for the sample TPU/POSS-OH(10). This peak can be ascribed to the melting of unbound POSS, as it not observable in the plots for samples which underwent a washing treatment to remove the unbound POSS.

In order to accurately evaluate the sample microphase morphology, TEM analysis was preformed. Figure 5 shows a comparison between TEM micrographs of the neat TPU and TPU/POSS-OH(5) surface which underwent the washing treatment.

It is clearly evident from this figure that the morphology of TPU/POSS-OH(5) does not show any segregation or aggregation of POSS particles, thus indicating that POSS-OH is chemically reacted with the polymer. Moreover, Si concentration has been found to be homogenous. A clear microphase-separated structure can be observed for both samples. However, the presence of POSS turns out to modify the microphase morphology, as neat TPU surface shows a finer structure compare to TPU/POSS$\mathrm{OH}(5)$. Indeed, the dimension of domains seems to increase in the POSS-based TPU, evidencing that

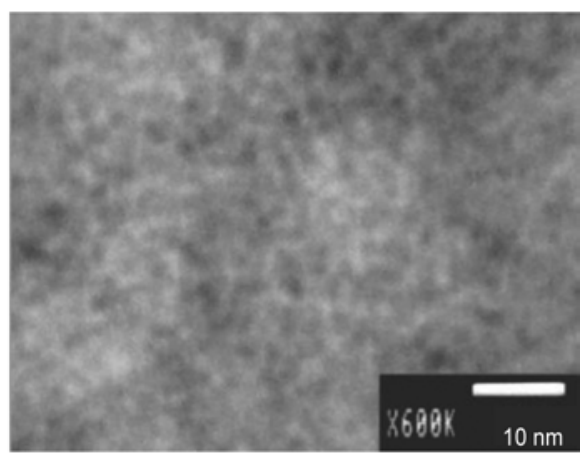

b)

Figure 5. TEM micrographs of: (a) TPU and (b) TPU/POSS-OH(5) 


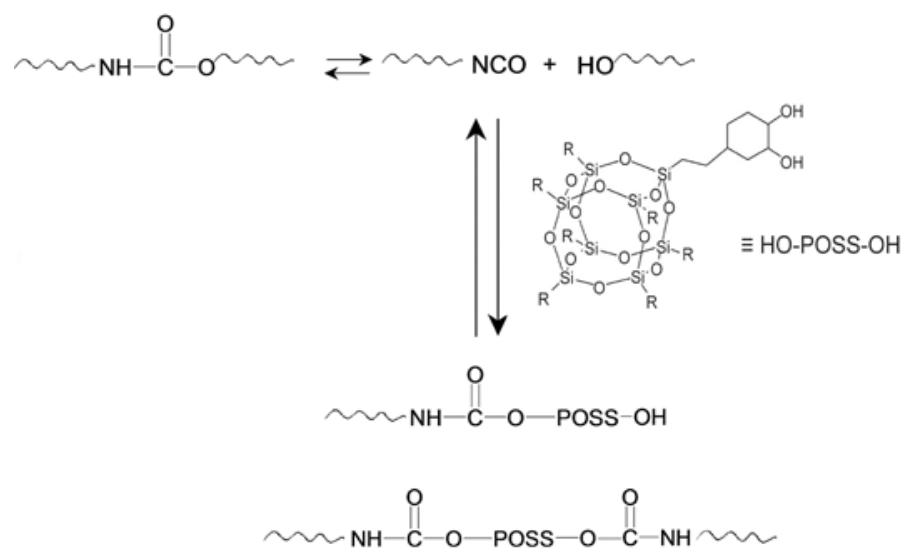

Figure 6. Reaction scheme

the silsesquioxane is capable of modifying the polymer microphase morphology, as previously reported in the literature [41]. On the basis of the results obtained, it is possible to hypothesize that the reaction between the polymer functional groups, formed during the melt blending, and POSS functionalities follows the mechanism shown in Figure 6.

Indeed, it is possible to suppose that both the silsesquioxane hydroxyl functionalities react with the polymer isocyanate groups, produced by chain scission, leading to POSS insertion inside the macromolecular chains or the reaction of only one of the two $\mathrm{OH}$ groups, forming POSS-ended chains. The effect of POSS as chain terminator is supported by the viscosity measurements, the relative viscosity of the pristine TPU and TPU/POSS-OH(10) being 1.41 and 1.21 , respectively.

Another property of the materials prepared, which was investigated by contact angle measurements, was the wettability. Indeed, water contact angle was measured on the surface of TPU/POSS-OH films up to $10 \%$ POSS, which underwent a washing treatment. While the contact angle of pristine TPU is about $95^{\circ}$, a strong reduction was obtained in the presence of POSS, to about 85 and $70^{\circ}$ for $5 \%$ and $10 \%$ POSS loadings, respectively. This can be explained by the hydrophilicity introduced by $\mathrm{OH}$ groups on POSS, which are present also in the hybrids because of the formation of POSS-ended chains characterized by a hydroxyl group.

\section{Conclusions}

In this work, a novel approach for the preparation of POSS-based hybrids has been assessed. The method studied turns out to be simple, being based on reactive melting, and it is applicable to polymers which do not carry reactive side groups. Indeed, the approach consists in the reaction between silsesquioxane molecules and the macromolecule functional groups, which are formed directly during the melt blending process through a controlled scission of the polymer matrix.

In the case of TPU, it has been verified that the thermal degradation of the urethane segment, which occurs directly in a mixer under controlled atmosphere by depolymerization leads to the formation of isocyanate groups. The blending with silsesquioxane molecules, characterized by hydroxyl groups, leads to the formation of systems consisting of silsesquioxane molecules directly attached to the macromolecular chain which modify the TPU microphase morphology. The presence of POSS has been found to affect significantly the polymer glass transition temperature, as it increases by increasing POSS concentration, this phenomenon being related to the effect of POSS on the macromolecular motion and to the partitioning of silsesquioxane molecules into the soft segment phase.

The presence of POSS has turned out to increase film hydrophilicity.

\section{Acknowledgements}

We are grateful to the Italian Ministry of Education and University through the 2010-2011 PRIN project (Grant No. 2010XLLNM3_005).

\section{References}

[1] Cordes D. B., Lickiss P. D., Rataboul F.: Recent developments in the chemistry of cubic polyhedral oligosilsesquioxanes. Chemical Reviews, 110, 2081-2173 (2010).

DOI: $10.1021 / \mathrm{cr} 900201 \mathrm{r}$

[2] Baney R. H., Itoh M., Sakakibara A., Suzuki T.: Silsesquioxanes. Chemical Reviews, 95, 1409-1430 (1995). DOI: $10.1021 / \mathrm{cr} 00037 \mathrm{a} 012$ 
[3] Valentini L., Bittolo Bon S., Monticelli O., Kenny J. M.: Deposition of amino-functionalized polyhedral oligomeric silsesquioxanes on graphene oxide sheets immobilized onto an amino-silane modified silicon surface. Journal of Materials Chemistry, 22, 6213 6217 (2012).

DOI: $10.1039 / \mathrm{C} 2 \mathrm{JM} 16111 \mathrm{G}$

[4] Blanco I., Abate L., Antonelli M. L., Bottino F. A., Bottino P.: Phenyl hepta cyclopentyl - polyhedral oligomeric silsesquioxane (ph,hcp-POSS)/polystyrene (PS) nanocomposites: The influence of substituents in the phenyl group on the thermal stability. Express Polymer Letters, 6, 997-1006 (2012).

DOI: $10.3144 /$ expresspolymlett.2012.105

[5] Carniato F., Bisio C., Gatti G., Boccaleri E., Bertinetti E., Coluccia S., Monticelli O., Marchese L.: Titanosilsesquioxanes embedded in synthetic clay as a hybrid material for polymer science. Angewandte Chemie International Edition, 48, 6059-6061 (2009).

DOI: $10.1002 /$ anie.200901927

[6] Tanaka K., Chujo Y.: Advanced functional materials based on polyhedral oligomeric silsesquioxane (POSS). Journal of Materials Chemistry, 22, 1733-1746 (2012). DOI: $10.1039 / \mathrm{C} 1 \mathrm{JM} 14231 \mathrm{C}$

[7] Baldi F., Bignotti F., Ricco L., Monticelli O., Riccò T.: Mechanical and structural characterization of POSSmodified polyamide 6. Journal of Applied Polymer Science, 100, 3409-3414 (2006).

DOI: $10.1002 /$ app.23804

[8] Dintcheva N. Tz., Morici E., Arrigo R., La Mantia F. P., Malatesta V., Schwab J. J.: Structure-properties relationships of polyhedral oligomeric silsesquioxane (POSS) filled PS nanocomposites. Express Polymer Letters, 6, 561-571 (2012).

DOI: $10.3144 /$ expresspolymlett.2012.59

[9] Vannier A., Duquesne S., Bourbigot S., Castrovinci A., Camino G., Delobel R.: The use of POSS as synergist in intumescent recycled poly(ethylene terephthalate). Polymer Degradation and Stability, 93, 818-826 (2008).

DOI: 10.1016/j.polymdegradstab.2008.01.016

[10] Phillips S. H., Haddad T. S., Tomczak S. J.: Developments in nanoscience: Polyhedral oligomeric silsesquioxane (POSS)-polymers. Current Opinion in Solid State and Materials Science, 8, 21-29 (2004).

DOI: $10.1016 / \mathrm{j} . \operatorname{cossms} .2004 .03 .002$

[11] Li G., Wang L., Ni H., Pittman C. U. Jr.: Polyhedral oligomeric silsesquioxane (POSS) polymers and copolymers: A review. Journal of Inorganic and Organometallic Polymers, 11, 123-154 (2001). DOI: 10.1023/A:1015287910502

[12] Monticelli O., Cavallo D., Bocchini S., Frache A., Carniato F., Tonelotto A.: A novel use of Ti-POSS as initiator of L-lactide ring-opening polymerization. Journal of Polymer Science Part A: Polymer Chemistry, 49, 4794-4799 (2011).

DOI: $10.1002 /$ pola. 24926
[13] Fina A., Tabuani D., Frache A., Camino G.: Polypropylene-polyhedral oligomeric silsesquioxanes (POSS) nanocomposites. Polymer, 46, 7855-7866 (2005).

DOI: $10.1016 /$ j.polymer.2005.06.121

[14] Fu B. X., Gelfer M. Y., Hsiao B. S., Phillips S., Viers B., Blanski R., Ruth P.: Physical gelation in ethylenepropylene copolymer melts induced by polyhedral oligomeric silsesquioxane (POSS) molecules. Polymer, 44, 1499-1506 (2003).

DOI: $10.1016 / \mathrm{S} 0032-3861(03) 00018-1$

[15] Hosaka N., Otsuka H., Hino M., Takahara A.: Control of dispersion state of silsesquioxane nanofillers for stabilization of polystyrene thin films. Langmuir, 24, 5766-5772 (2008).

DOI: $10.1021 / 1 a 704062 \mathrm{n}$

[16] Hao N., Böhning M., Schönhals A.: Dielectric properties of nanocomposites based on polystyrene and polyhedral oligomeric phenethyl-silsesquioxanes. Macromolecules, 40, 9672-9679 (2007).

DOI: $10.1021 / \mathrm{ma} 071777 \mathrm{~g}$

[17] Monticelli O., Fina A., Cozza E. S., Prato M., Bruzzo V.: POSS vapor phase grafting: A novel method to modify polymer films. Journal of Materials Chemistry, 21, 18049-18054 (2011).

DOI: $10.1039 / \mathrm{C} 1 \mathrm{JM} 13553 \mathrm{H}$

[18] Fina A., Monticelli O., Camino G.: POSS-based hybrids by melt/reactive blending. Journal of Materials Chemistry, 20, 9297-9305 (2010). DOI: 10.1039/C0JM00480D

[19] Monticelli O., Fina A., Ullah A., Waghmare P.: Preparation, characterization, and properties of novel PSMAPOSS systems by reactive blending. Macromolecules, 42, 6614-6623 (2009).

DOI: $10.1021 / \mathrm{ma900969b}$

[20] Kim B-S., Mather P. T.: Amphiphilic telechelics incorporating polyhedral oligosilsesquioxane: 1 . Synthesis and characterization. Macromolecules, 35, 8378-8384 (2002).

DOI: $10.1021 / \mathrm{ma} 020226 \mathrm{~h}$

[21] Kim B-S., Mather P. T.: Amphiphilic telechelics with polyhedral oligosilsesquioxane (POSS) end-groups: Dilute solution viscometry. Polymer, 47, 6202-6207 (2006).

DOI: $10.1016 /$ j.polymer.2006.06.050

[22] Dvornic P. R., Hartmann-Thompson C., Keinath S. E., Hill E. J.: Organic-inorganic polyamidoamine (PAMAM) dendrimer-polyhedral oligosilsesquioxane (POSS) nanohybrids. Macromolecules, 37, 7818-7831 (2004). DOI: $10.1021 / \mathrm{ma} 030542 \mathrm{~b}$

[23] Fu B. X., Zhang W., Hsiao B. S., Rafailovich M., Sokolov J., Johansson G., Sauer B. B., Phillips S., Balnski R.: Synthesis and characterization of segmented polyurethanes containing polyhedral oligomeric silsesquioxanes nanostructured molecules. High Performance Polymer, 12, 565-571 (2000). DOI: $10.1088 / 0954-0083 / 12 / 4 / 311$ 
[24] Fu B. X., Hsiao B. S., White H., Rafailovich M., Mather P. T., Jeon H. G., Phillips S., Lichtenhan J., Schwab J.: Nanoscale reinforcement of polyhedral oligomeric silsesquioxane (POSS) in polyurethane elastomer. Polymer International, 49, 437-440 (2000). DOI: $10.1002 /(\mathrm{SICI}) 1097-0126(200005) 49: 5<437:$ : AID-PI239>3.0.CO;2-1

[25] Fu B. X., Hsiao B. S., Pagola S., Stephens P., White H., Rafailovich M., Sokolov J., Mather P. T., Jeon H. G., Phillips S., Lichtenhan J., Schwab J.: Structural development during deformation of polyurethane containing polyhedral oligomeric silsesquioxanes (POSS) molecules. Polymer, 42, 599-611 (2001).

DOI: 10.1016/S0032-3861(00)00389-X

[26] Turri S., Levi M.: Structure, structure, dynamic properties, and surface behavior of nanostructured ionomeric polyurethanes from reactive polyhedral oligomeric silsesquioxanes. Macromolecules, 38, 5569-5574 (2005).

DOI: $10.1021 / \mathrm{ma} 047304 \mathrm{~g}$

[27] Knight P. T., Lee K. M., Qin H., Mather P. T.: Biodegradable thermoplastic polyurethanes incorporating polyhedral oligosilsesquioxane. Biomacromolecules, 9, 2458-2467 (2008). DOI: $10.1021 / \mathrm{bm} 8004935$

[28] Wu J., Ge Q., Mather P. T.: PEG-POSS multiblock polyurethanes: Synthesis, characterization, and hydrogel formation. Macromolecules, 43, 7637-7649 (2010). DOI: $10.1021 / \mathrm{ma101336 \textrm {c }}$

[29] Nanda A. K., Wicks D. A., Madbouly S. A., Otaigbe J. U.: Nanostructured polyurethane/POSS hybrid aqueous dispersions prepared by homogeneous solution polymerization. Macromolecules, 39, 7037-7043 (2006).

DOI: $10.1021 / \mathrm{ma} 060809 \mathrm{~h}$

[30] Oaten M., Choudhury N. R.: Silsesquioxane-urethane hybrid for thin film applications. Macromolecules, 38, 6392-6401 (2005). DOI: $10.1021 / \mathrm{ma} 0476543$

[31] Neumann D., Fisher M., Tran L., Matisons J. G.: Synthesis and characterization of an isocyanate functionalized polyhedral oligosilsesquioxane and the subsequent formation of an organic-inorganic hybrid polyurethane. Journal of the American Chemical Society, 124, 13998-13999 (2002).

DOI: $10.1021 /$ ja0275921
[32] Liu H., Zheng S.: Polyurethane networks nanoreinforced by polyhedral oligomeric silsesquioxane. Macromolecular Rapid Communication, 26, 196-200 (2005). DOI: $10.1002 /$ marc.200400465

[33] Bourbigot S., Turf T., Bellayer S., Duquesne S.: Polyhedral oligomeric silsesquioxane as flame retardant for thermoplastic polyurethane. Polymer Degradation and Stability, 94, 1230-1237 (2009).

DOI: $10.1016 /$ j.polymdegradstab.2009.04.016

[34] Chattopadhyay D. K., Webster D. C.: Thermal stability and flame retardancy of polyurethanes. Progress in Polymer Science, 34, 1068-1133 (2009).

DOI: $10.1016 /$ j.progpolymsci.2009.06.002

[35] Silverstein R. M., Webster F. X., Klemie D. J.: Spectrometric identification of organic compounds. Wiley, New York (2005).

[36] Stuart B.: Infrared spectroscopy. Fundamental and applications. Wiley, New York (2004).

[37] Lin-Vien D., Colthup N. B., Fateley W. G., Grasselli J. G.: The handbook of infrared and Raman characteristic frequencies of organic molecules. Academic Press, Boston (1991).

[38] Bharadwaj R. K., Berry R. J., Farmer B. L.: Molecular dynamics simulation study of norbornene-POSS polymers. Polymer, 41, 7209-7221 (2000). DOI: 10.1016/S0032-3861(00)00072-0

[39] Lee A., Lichtenhan J. D.: Viscoelastic responses of polyhedral oligosilsesquioxane reinforced epoxy systems. Macromolecules, 31, 4970-4974 (1998). DOI: $10.1021 / \mathrm{ma} 9800764$

[40] Raftopoulos K. N., Pandis Ch., Apekis L., Pissis P., Janowski B., Pielichowski K., Jaczewska J.: Polyurethane-POSS hybrids: Molecular dynamics studies. Polymer, 51, 709-718 (2010). DOI: 10.1016/j.polymer.2009.11.067

[41] Madbouly S. A., Otaigbe J. U., Nanda A. K., Wicks D. A.: Rheological behavior of POSS/polyurethane-urea nanocomposite films prepared by homogeneous solution polymerization in aqueous dispersions. Macromolecules, 40, 4982-4991 (2007).

DOI: $10.1021 / \mathrm{ma} 070186 n$ 